\title{
An indirect technique in nuclear astrophysics: alpha- cluster transfer reaction
}

\author{
Yang-Ping Shen ${ }^{1, *}$, Bing Guo ${ }^{1}$, and Wei-Ping Liu ${ }^{1}$ \\ ${ }^{1}$ China Institute of Atomic Energy, P.O. Box 275 (10), Beijing 102413, China
}

\begin{abstract}
Helium $\left({ }^{4} \mathrm{He}\right.$, or $\left.\alpha\right)$ is the second most abundant element in the observable Universe. The $\alpha$-particle induced reactions such as $(\alpha, \gamma),(\alpha, \mathrm{n})$ and $(\alpha, \mathrm{p})$ play a crucial role in nuclear astrophysics, especially for understanding stellar helium burning. Because of the strong Coulomb repulsion, it is greatly hindered to directly measure the cross sections for these $\alpha$-capture reactions at stellar energies. Alpha-cluster transfer reaction is a powerful tool for investigation of astrophysical $(\alpha, \gamma),(\alpha, \mathrm{n})$ and $(\alpha, \mathrm{p})$ reactions since it can preferentially populate the natural-parity states with an $\alpha$-cluster structure which dominantly contribute to these astrophysical $\alpha$-capture reactions during stellar helium burning. In this paper, we review the theoretical scheme, the experimental technique, astrophysical applications and the future perspectives of such approach based on $\alpha$-cluster transfer reactions.
\end{abstract}

\section{Introduction}

Nuclear astrophysics research is currently a frontier in the quest to understand how the elements in Universe were created and how stars evolve over time. Thousands of nuclear processes are responsible for the synthesis of the elements and drive the evolution of stars. These nuclear reactions are mostly triggered by hydrogen, helium, and heavy ions. Apart from the major so-called Big Bang Nucleosynthesis (BBN) [1,2], the ${ }^{4} \mathrm{He}$ nuclide (namely, $\alpha$ cluster or $\alpha$ particle) can also be created during the hydrogen-burning phase within stars [3], which did not occur until about 100 million years after the Big Bang. Because ${ }^{4} \mathrm{He}$ is the second most abundant element in the observable Universe after hydrogen, $\alpha$-cluster induced reactions like $(\alpha, \gamma),(\alpha, \mathrm{n})$, and $(\alpha, \mathrm{p})$ play a crucial role in nuclear astrophysics, especially for understanding stellar helium burning, which is a critical stage during the evolution of stars.

Because the energies corresponding to typical temperatures in stars are significantly below the high Coulomb barrier, the direct measurements of $\alpha$-particle induced reactions at stellar energies (so-called Gamow window) are greatly hindered due to the vanishing cross section resulting from the small Coulomb penetrability at energies of astrophysical interest. The ${ }^{12} \mathrm{C}(\alpha, \gamma){ }^{16} \mathrm{O}$ cross section, for example, is expected to be on the order of $10^{-17} \mathrm{~b}$ at 300 $\mathrm{keV}$ corresponding to the average temperature of helium burning. By far, this is more than five orders of magnitude lower than the maximum sensitivity achieved by the most advanced measurements. Generally the estimations of the cross sections at stellar energies are extrapolated from experimental data with a much higher energy range. However, because unknown

\footnotetext{
*e-mail: fermi09@foxmail.com
} 
resonant states offen exist in the energy range of astrophysical interest, and free parameters in extrapolations are not able to be properly constrained by data at high energies. Therefore, such extrapolations may lead to considerable uncertainty. As a result, indirect approaches (for example, $\alpha$-cluster transfer reactions) are particularly useful for reactions that are difficult, if not impossible, to measure directly. These techniques can be used to derive level parameters (e.g., energies, asymptotic normalization coefficients (ANC) or spectroscopic factors (SFs), lifetimes) that can then be used in the analysis of the $R$-matrix or other reaction model [4].

Several indirect techniques for studying astrophysical $\alpha$-capture reactions have been developed and implemented. In present article, we focuse on a specific and complementary method, $\alpha$-cluster transfer reactions, which specially aims at determining the cross sections and the stellar rates of $\alpha$-particle induced reactions in the stellar helium burning phase, which are more difficult directly to measure at the Gamow window than proton induced reactions since they have higher Coulomb barrier. Because the $\alpha$ transfer reaction is most likely to occur by $\alpha$-cluster transfer mechanism, it can not only be used to study $\alpha$-particle induced astrophysical reactions [5], but also to evaluate the nuclear structure (e.g., $\alpha$-decay widths [6]) and nuclear reaction mechanisms [7].

\section{DWBA theory}

In reactions of type $A(a, b) B$, the nuclei $\mathrm{A}$ and a usually start in their ground states. In general, direct reaction and compound-nucleus reaction mechanisms are used to describe transfer reactions. Only a few nucleons or the nucleus as a whole on the surface of the nucleus are involved in the reactions that proceed most quickly. They are called direct reactions, and usually occur at high incident energies. This is because such reactions finish more quickly, leading to fewer internal collisions. Because the final nuclei's directions are significantly more impacted by the initial direction in these quick reactions, they often exhibit high cross sections at forward angles.

The DWBA theory, which assumes a one-step transition between the initial and final scattering states, is widely applied to model the direct reactions. This theory proves very useful in description of such reactions. Of course, the compound-nucleus reaction process is still viable at lower energies or at backward angles. However, this mechanism produces isotropic angular distributions which can be easily distinguished from forward-peaked cross sections. It can also be evaluated and, if necessary, subtracted. In addition one-step assumption may be improved by including two and higher-order steps, as in a perturbation series. Coupledchannels approaches must be employed in this scenario. More details about the Higher-order effects analysis can be found in Ref. [8].

In the DWBA theory, the most important equation is the relation between the reduced DWBA and experimental differencial cross sections. Considering the $\alpha$-cluster transfer reaction $A+a \rightarrow b+B$, the relation is given to be

$$
\left(\frac{d \sigma}{d \Omega}\right)_{\exp }=\sum_{l_{B} j_{B} l_{a} j_{a}} S_{A \alpha l_{B} j_{B}} S_{b \alpha l_{a} j_{a}} \sigma_{l_{B} j_{B} l_{a} j_{a}}^{\mathrm{DWBA}},
$$

where $\sigma_{l_{B} j_{B} l_{a} j_{a}}^{\mathrm{DWBA}}$ denotes the reduced DWBA cross section. In addition the asymptotic normalization coefficient (ANC) can be related to the spectroscopic factor by

$$
\left(C_{\beta \gamma l_{\varphi} j_{\varphi}}\right)^{2}=S_{\beta \gamma l_{\varphi} j_{\varphi}} \cdot b_{\beta \gamma l_{\varphi} j_{\varphi}}^{2} .
$$

$\sigma_{l_{B} j_{B} l_{a} j_{a}}^{\mathrm{DWBA}}$ depend on the optical model potential (OMP) parameters for the initial $(a+A)$ and final $(b+B)$ channels and the real binding potentials for the initial $(a)$ and final nuclei 
(B). Usually the OMP parameters of the initial and final channels can be fixed by fitting the experimental angular distributions of elastic scattering for the initial and final channels. The geometrical parameters for the binding potentials can be constrained by a minimum- $\chi^{2}$ fitting to the experimental data of transfer reaction angular distributions. Another typical method to constrain the binding potential is to reproduce the root-mean-square (rms) radius of the $\alpha$-cluster wave function (e.g., for the $B=(A \alpha)$ system) using the formula [9]

$$
\left\langle r_{B}^{2}\right\rangle=\frac{m_{\mathrm{He}}}{m_{B}}\left\langle r_{\mathrm{He}}^{2}\right\rangle+\frac{m_{A}}{m_{B}}\left\langle r_{A}^{2}\right\rangle+\frac{m_{\mathrm{He}} m_{A}}{m_{B}^{2}}\left\langle r^{2}\right\rangle .
$$

Here $\left\langle r_{B}^{2}\right\rangle,\left\langle r_{\mathrm{He}}^{2}\right\rangle$, and $\left\langle r_{A}^{2}\right\rangle$ are the the rms radii of the compound nucleus $B$, the valence cluster $\mathrm{He}$, and the core $A$, which can be determined experimentally or theoretically. $\left\langle r^{2}\right\rangle$ is the root-mean-square (rms) radius of the $\alpha$-cluster wave function.

\section{Experimental techniques}

From the perspective of nuclear astrophysics, $\alpha$ transfer reactions with large cross sections provide an alternative method for extracting level parameters such as the $\alpha$ spectroscopic factor $\left(S_{\alpha}\right)$, spectroscopic amplitude (SA), ANCs, or reduced $\alpha$ widths for the subthreshold resonances crucially involved in determining the astrophysical S-factor of the challenging $\alpha$-capture reactions. Thus, in studies involving $\alpha$-transfer processes, the selection of the $\alpha$ transfer systems is the highest priority to consider. The most common transfer systems are the $\left({ }^{6} \mathrm{Li}, \mathrm{d}\right)$ and $\left({ }^{7} \mathrm{Li}, \mathrm{t}\right)$ reactions. The $\left({ }^{11} \mathrm{~B},{ }^{7} \mathrm{Li}\right)$ reaction has also been proposed as a suitable transfer system for studying cluster structures and astrophysical reactions in recent years.

In the DWBA calculations, the $S_{\alpha}$ or the ANC of the $\alpha$ provider in the transfer systems, such as ${ }^{6} \mathrm{Li}$ in the $\left({ }^{6} \mathrm{Li}, \mathrm{d}\right)$ system, ${ }^{7} \mathrm{Li}$ in the $\left({ }^{7} \mathrm{Li}, \mathrm{t}\right)$ system and ${ }^{11} \mathrm{~B}$ in the $\left({ }^{11} \mathrm{~B},{ }^{7} \mathrm{Li}\right)$ system, is necessarily required to derive the desirable level parameters. The detailed table of $S_{\alpha} / \mathrm{SA}$ and ANC results of the ${ }^{6,7} \mathrm{Li}$ and ${ }^{11} \mathrm{~B}$ ground states for the $\left({ }^{6} \mathrm{Li}, \mathrm{d}\right),\left({ }^{7} \mathrm{Li}, \mathrm{t}\right)$ and $\left({ }^{11} \mathrm{~B},{ }^{7} \mathrm{Li}\right)$ transfer systems can be found in Ref. [10].

In addition to $\left({ }^{6} \mathrm{Li}, \mathrm{d}\right),\left({ }^{7} \mathrm{Li}, \mathrm{t}\right)$ and $\left({ }^{11} \mathrm{~B},{ }^{7} \mathrm{Li}\right)$, other transfer systems, such as $\left({ }^{16} \mathrm{O},{ }^{12} \mathrm{C}\right)$ and $\left({ }^{20} \mathrm{Ne},{ }^{16} \mathrm{O}\right)$, also have potential for investigating $\alpha$-cluster structure and $\alpha$ induced reactions of astrophysical interest. To date the ANC of the ${ }^{16} \mathrm{O}$ GS has been extensively investigated. There is a large discrepancy of up to a factor 240 between these reported experimental values of the GS ANC ranging from $13.9 \pm 2.4 \mathrm{fm}^{-1 / 2}$ to $3390 \mathrm{fm}^{-1 / 2}$ [11-15]. Although the most recent measurement by Shen et al. [15] gives a more precise value, further highprecision measurements of the ${ }^{16} \mathrm{O}$ GS ANC are still desirable. This is because the ${ }^{16} \mathrm{O}$ GS ANC is crucial for restricting the GS external capture in the ${ }^{12} \mathrm{C}(\alpha, \gamma){ }^{16} \mathrm{O}$ reaction, as well as for studying astrophysical reactions as a powerful tool. As for the ${ }^{20} \mathrm{Ne}$ GS ANC, although Motobayashi et al. [16] presented the first experimental determination through the elastic transfer reaction ${ }^{20} \mathrm{Ne}\left({ }^{16} \mathrm{O},{ }^{20} \mathrm{Ne}\right){ }^{16} \mathrm{O}$ using $\Delta E$ - $E$ silicon detector telescope, it is still necessary to perform high-precision measurement of the ${ }^{20} \mathrm{Ne}$ GS ANC because of its potential application to astrophysical reactions.

With the transfer systems introduced above, here we discuss three kinds of typical experiments, including sub-Coulomb measurement of $\alpha$-transfer reactions, high-energy measurement of $\alpha$-transfer reactions, and high-resolution coincidence measurement of absolute $\alpha$-decay widths.

Performing transfer reaction measurements at sub-Coulomb energies is challenging experimentally, because the cross sections become rather small and also the energies of the recoil nuclei are low. However, such measurements are crucial for reducing the dependence 
of the results on optical potential parameters and the influence of compound-nuclear contributions. This is because, at sub-Coulomb energies, DWBA calculations are primarily governed by Coulomb potentials for both the exit and entrance channels, and seldom depend on nuclear potential parameters. The DWBA cross sections are thus essentially model independent. The pioneering sub-Coulomb measurements of the ${ }^{12} \mathrm{C}\left({ }^{6} \mathrm{Li}, \mathrm{d}\right){ }^{16} \mathrm{O}$ and ${ }^{12} \mathrm{C}\left({ }^{7} \mathrm{Li}, \mathrm{t}\right){ }^{16} \mathrm{O}$ cross sections were performed by Brune et al. [17] to investigate the ${ }^{12} \mathrm{C}(\alpha, \gamma){ }^{16} \mathrm{O}$ S-factor by determining the reduced $\alpha$ widths of the $2^{+}$and $1^{-}$subthreshold states in ${ }^{16} \mathrm{O}$. The normalized DWBA calculations well reproduced the experimental excitation functions for both ${ }^{12} \mathrm{C}\left({ }^{6} \mathrm{Li}, \mathrm{d}\right){ }^{16} \mathrm{O}$ and ${ }^{12} \mathrm{C}\left({ }^{7} \mathrm{Li}, \mathrm{t}\right){ }^{16} \mathrm{O}$ leading to the $2^{+}$and $1^{-}$states of ${ }^{16} \mathrm{O}$.

An alternative technique is sub-Coulomb measurement of transfer-reaction differential cross sections as a function of angle, namely, angular distributions. For example, the astrophysical S-factor of the ${ }^{13} \mathrm{C}(\alpha, \mathrm{n}){ }^{16} \mathrm{O}$ reaction was investigated by determining the ANC for the $6356 \mathrm{keV} 1 / 2^{+}$state of ${ }^{17} \mathrm{O}$ using the ${ }^{13} \mathrm{C}\left({ }^{6} \mathrm{Li}, \mathrm{d}\right){ }^{17} \mathrm{O}$ reaction in inverse kinematics, performed at two sub-Coulomb energies $[18,19]$. It should be noted that the technique based on excitation function measurement is not suitable for a high-energy measurement because in that case compound-nucleus contribution and higher-order effects can no longer be ignored, causing DWBA calculations to fail to reasonably reproduce experimental total cross sections.

Comparing with Sub-Coulomb transfer measurement, the high-energy transfer measurement allows one to use a high-precision magnetic spectrograph for resolving the closely spaced levels which cannot be achieved only by silicon detectors in sub-Coulomb transfer measurement. Therefore, these two techniques can be used complementarily to study $\alpha$-cluster structures and then astrophysical nuclear reactions. In Fig. 1 we schematically show a typical experimental setup for high-energy transfer measurement performed using the Q3D magnetic spectrograph at HI-13 tandem accelerator of China Institute of Atomic Energy (CIAE). The reaction products were separated and focused by the magnetic spectrograph and detected by two-dimensional position sensitive silicon detector array fixed at the focal plane. The two-dimensional position information enables the products emitted into the acceptable solid angle to be completely recorded, and the energy information was used to remove the impurities with the same magnetic rigidity, as discussed in Refs. [9, 15, 20]. Typically the Q3D magnetic spectrograph has an energy resolution of $0.02 \%$ and an angular resolution of 0.1 degree, which makes it possible to resolve the closely spaced states which cannot be achieved only by silicon detectors, and to obtain high-precision data of angular distributions at forward angles where direct mechanism dominates. High-precision data of the angular distributions at forward angles are helpful for constraining the binding potential parameters with the minimum $\chi^{2}$ fitting.

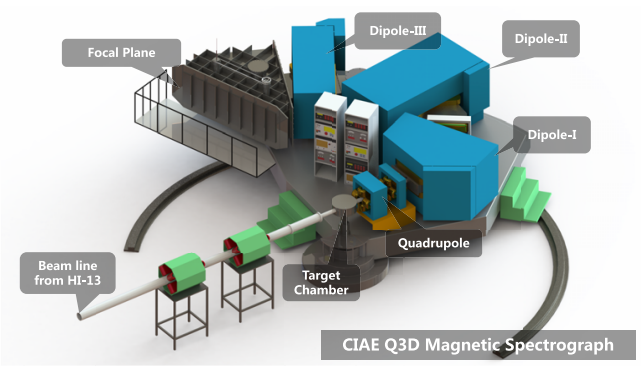

Figure 1. Typical experimental setup for high-energy transfer measurement performed using the Q3D magnetic spectrograph at HI-13 tandem accelerator of CIAE. The Q3D magnetic spectrograph consists of a target chamber, a quadrupole, three dipoles, and detector arrays at the focal plane. 
As for the resonances far above the $\alpha$-decay threshold, large $\alpha$-decay widths of these resonances allow us to perform high-resolution coincidence measurement of absolute $\alpha$-decay widths. For example, Wheldon et al. [21] unambiguously measured the absolute $\alpha$-decay widths from the excited states in ${ }^{16} \mathrm{O}$ within the energy range 13.85 to $15.87 \mathrm{MeV}$ through the ${ }^{12} \mathrm{C}\left({ }^{6} \mathrm{Li}, \mathrm{d}\right){ }^{16} \mathrm{O}$ reaction by using a large-acceptance position-sensitive silicon detector array placed near the target position in coincidence with the high-resolution Q3D magnetic spectrograph. The deuteron ejectiles were analyzed by the Q3D spectrograph, while the recoil or the breakup products were recorded by the silicon array comprised of four double-sided silicon-strip detectors. Such a coincidence measurement technique is useful to investigate the resonances with considerable $\alpha$ widths. Similar application of this technique has been achieved in other astrophysical reactions such as the ${ }^{15} \mathrm{O}(\alpha, \gamma){ }^{19} \mathrm{Ne}$ reaction (see, e.g., Ref. [22]).

\section{$4{ }^{12} \mathrm{C}(\alpha, \gamma){ }^{16} \mathrm{O}$, a example for the application of the transfer reaction method}

The ${ }^{12} \mathrm{C}(\alpha, \gamma){ }^{16} \mathrm{O}$ reaction, which is one of the most important reactions in nuclear astrophysics, is dominated by multiple supra- and sub-threshold resonance to ${ }^{16} \mathrm{O}$ excited states. The complicated reaction mechanism in ${ }^{12} \mathrm{C}(\alpha, \gamma){ }^{16} \mathrm{O}$ provides an excellent case for the application of indirect techniques, especially the transfer reaction method. The resonance to the

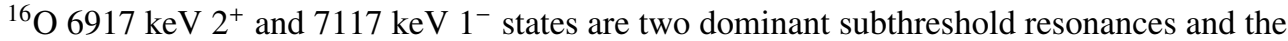
external capture to the ${ }^{16} \mathrm{O}$ ground state is also expected to be unneglectable. Many works with $\left({ }^{6} \mathrm{Li}, \mathrm{d}\right),\left({ }^{7} \mathrm{Li}, \mathrm{t}\right)$ and $\left({ }^{11} \mathrm{~B},{ }^{7} \mathrm{Li}\right)$ transfer reactions are reported. We recently measured the ANC of the ${ }^{16} \mathrm{O} 6917 \mathrm{keV} 2^{+}$and ground states with ${ }^{12} \mathrm{C}\left({ }^{11} \mathrm{~B},{ }^{7} \mathrm{Li}\right){ }^{16} \mathrm{O}$ transfer reaction [15, 23]. A comparison of $S_{E 2}(300)$ is shown in Fig. 2.

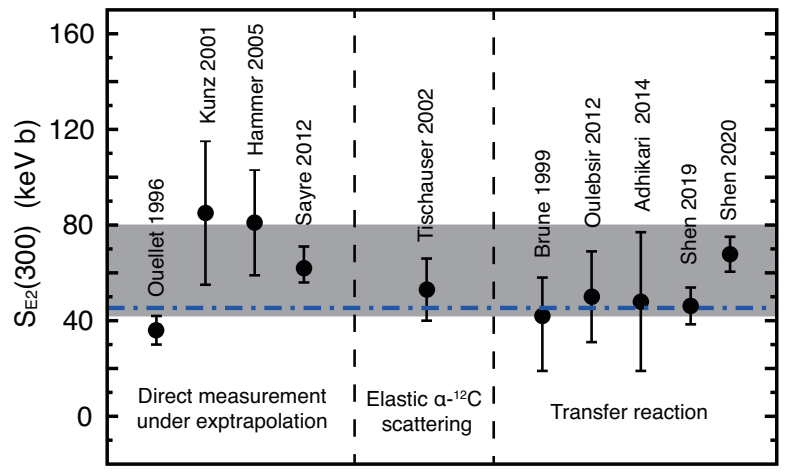

Figure 2. The $S_{E 2}(300)$ comparison $[11,15,17,23-29]$. The grey shadow represents the compilation value of NACRE II (2013) [30]. The blue dot-dashed line is the value in deBoer et al. (2017) [4].

It's seen that the $S_{E 2}$ factors given by the transfer reaction method are in good agreement with the values given by direct measurements and other indirect methods like elastic scattering. And the results with different transfer systems are also consistent with each other, which proves the systematic uncertainty from the diversity of different transfer systems is under control. One thing that should be mentioned is that the $S_{E 2}$ factor given by Shen et al. (2020) [15] is significantly higher than others. This is because the contribution from the external capture to the ${ }^{16} \mathrm{O}$ ground state is considered and found to be large in Shen et al. (2020) [15]. 


\section{Discussion and outlook}

The alpha-cluster transfer reaction has long been a powerful tool for determining the level parameters of the unknown resonant states with an $\alpha$-cluster structure and further investigating astrophysical $(\alpha, \gamma),(\alpha, \mathrm{n})$ and $(\alpha, \mathrm{p})$ reactions during helium burning phase of stars, and it continues to be so in the latest state-of-the-art analyses. To date this technique has been unambiguously verified by reproducing the well-known width, and has been extensively used to investigate astrophysical $\alpha$-particle induced reactions on stable nuclei such as the main neutron source reaction ${ }^{13} \mathrm{C}(\alpha, \mathrm{n}){ }^{16} \mathrm{O}$ of the $s$-process nucleosynthesis and the so-called "holy grail" reaction ${ }^{12} \mathrm{C}(\alpha, \gamma){ }^{16} \mathrm{O}$, as well as reactions on long-lived unstable nuclei, such as the ${ }^{14} \mathrm{C}(\alpha, \gamma){ }^{18} \mathrm{O}$ reaction.

As an indirect technique, it turns out to be one of the most useful methods in determining the contributions from the subthreshold states that are extremely difficult to constrain using the high-energy cross sections data from direct measurements, since the data at high energies have no effective constraints on such contributions while low-energy measurements are greatly hindered by the Coulomb barrier. Therefore, indirect techniques will continue to be highly desirable in the future, in addition to development of novel or underground direct measurements.

\section{Acknowledgments}

We would like to thank our colleagues of nuclear astrophysics team, without whom the results reviewed in this article would not have been achieved. This work is supported by the National Natural Science Foundation of China under Grants No. 12125509, 12005304, 11490561, U1867214, 11961141003.

\section{References}

[1] B.D. Fields, Annu. Rev. Nucl. Part. Sci. 61, 47 (2011)

[2] R.H. Cyburt, et al., Rev. Mod. Phys. 88, 015004 (2016)

[3] E.M. Burbidge, et al., Rev. Mod. Phys. 29, 547 (1957)

[4] R.J. deBoer, et al., Rev. Mod. Phys. 89, 035007 (2017)

[5] L. Buchmann, et al., Astrophys. J. 324, 953 (1988)

[6] D.F. Jackson, M. Rhoades-Brown, Nature 267, 593 (1977)

[7] P. Hodgson, E. Beták, Phys. Rep. 374, 1 (2003)

[8] I.J. Thompson, F.M. Nunes, Cambridge University Press, 2009 (2009)

[9] B. Guo, et al., Astrophys. J. 756, 193 (2012)

[10] Y.P. Shen, B. Guo, W.P. Liu, Prog. Part. Nucl. Phys. 119, 103857 (2021)

[11] D.B. Sayre, et al., Phys. Rev. Lett. 109, 142501 (2012)

[12] S. Adhikari, C. Basu, Phys. Lett. B 682, 216 (2009)

[13] S. Adhikari, et al., J. Phys. G 44, 015102 (2017)

[14] M.C. Morais, R. Lichtenthäler, Nucl. Phys. A 857, 1 (2011)

[15] Y.P. Shen, et al., Phys. Rev. Lett. 124, 162701 (2020)

[16] T. Motobayashi, et al., Nucl. Phys. A 331, 193 (1979)

[17] C.R. Brune, et al., Phys. Rev. Lett. 83, 4025 (1999)

[18] E.D. Johnson, et al., Phys. Rev. Lett. 97, 192701 (2006)

[19] M.L. Avila, et al., Phys. Rev. C 91, 048801 (2015)

[20] Y.P. Shen, et al., Phys. Lett. B 797, 134820 (2019) 
[21] C. Wheldon, et al., Phys. Rev. C 83, 064324 (2011)

[22] W.P. Tan, et al., Phys. Rev. Lett. 98, 242503 (2007)

[23] Y.P. Shen, et al., Phys. Rev. C 99, 025805 (2019)

[24] J.M.L. Ouellet, et al., Phys. Rev. C 54, 1982 (1996)

[25] R. Kunz, et al., Phys. Rev. Lett. 86, 3244 (2001)

[26] J.W. Hammer, et al., Nucl. Phys. A 752, 514 (2005)

[27] P. Tischhauser, et al., Phys. Rev. Lett. 88, 072501 (2002)

[28] N. Oulebsir, et al., Phys. Rev. C 85, 035804 (2012)

[29] S. Adhikari, et al., Phys. Rev. C 89, 044618 (2014)

[30] Y. Xu, et al., Nucl. Phys. A 918, 61 (2013) 